\title{
Gene therapy before the cradle
}

Neuronopathic Gaucher disease (nGD) is a lysosomal storage disorder caused by a deficiency in the gene that encodes glucosylceramidase (GBA). In adult patients, the mild form of the disease - which presents mainly with hepatomegaly and splenomegaly can be managed by enzyme replacement therapy. In infants, however, the acute form of nGD is untreatable as the enzyme cannot cross the bloodbrain barrier and children generally die within the first two years. Now, writing in Nature Medicine, Simon Waddington and colleagues have prevented development of nGD in mice fetuses by restoring expression of GBA through intracranial delivery of the gene in utero.

After having worked in fetal gene therapy in haemophilia, Waddington decided to pursue fetal gene therapy for neurodegenerative diseases. "We wanted to choose a neurological disease that was really severe so that, if we could reach it with gene therapy, it would then inspire our colleagues to adopt the technology," explains Waddington. The mouse model for nGD could replicate the human disease well, so the team decided to focus on this challenging disease.

The authors first intracranially injected an adeno-associated virus serotype 9 (AAV9) vector encoding green fluorescent protein (GFP) and observed homogeneity of expression of GFP, which extended from the prefrontal cortex to the cerebellum and brainstem. Next, using the same vector and timing of injection, they delivered human AAV9-GBA as a transuterine injection into the brains of knockout pups of $G b a$

\section{cif \\ fetal gene \\ therapy is \\ technically \\ possible (when \\ medically \\ justifiable) and}

maybe even an

improvement

on postnatal

gene therapy

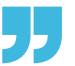

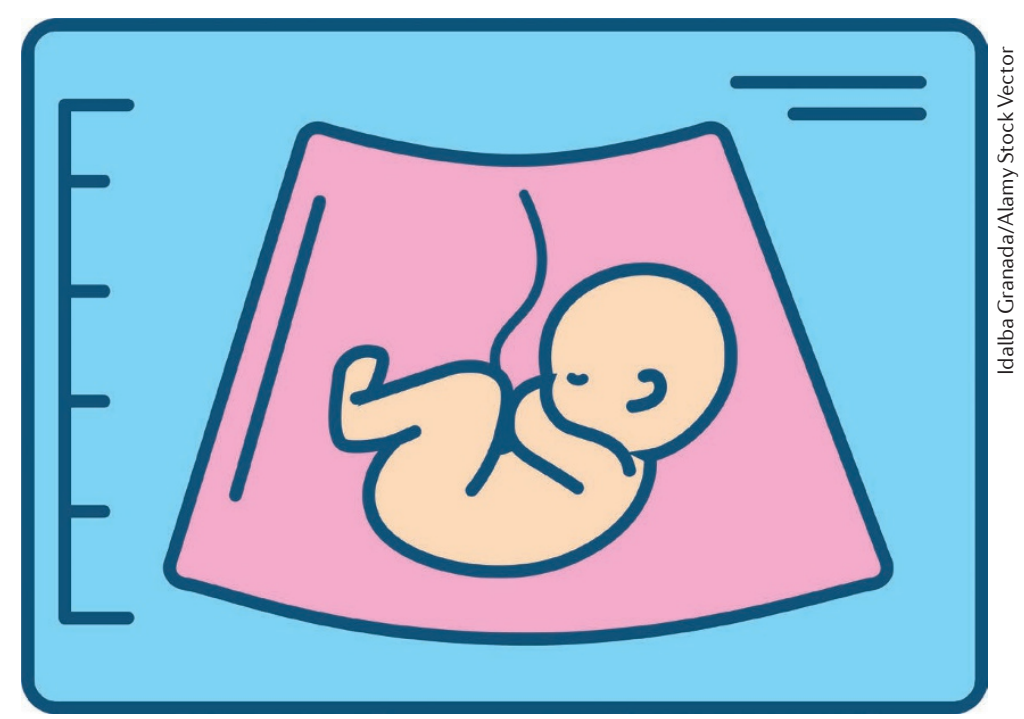

heterozygous parents. Once born, all treated knockouts seemed normal and fertile, and two of the mice were able to produce a litter of two symptomatic knockouts, whereas untreated control knockouts developed paralysis. Gene therapy prevented neuronal loss in knockouts in the thalamus, and restored normal expression of GBA in cortical layers. However, mice still developed visceral disease.

Because intravenous AAV9 can cross the blood-brain barrier of neonatal mice, the authors then tested both intravenous and intracranial administration of AAV9-GBA in newborn (P0-P1) knockouts to test whether this approach could ameliorate both visceral and brain pathology. Both cohorts were rescued from neonatal death and showed no paralysis or any major significant differences in motor skills compared with the wildtype mice. Intravenous gene therapy normalized enzyme activity in all visceral organs, except the heart, and prevented infiltration of Gaucher cells in spleen, liver and lungs, whereas intracerebroventricular injection failed to prevent Gaucher cell infiltration. However, the intravenous injection was not as effective in correcting the neurological inflammation and the mice had brain damage. Therefore, the intracerebroventricular gene therapy is more effective in treating the lethal neuropathology (although not as effective as the fetal injection) than the intravenous administration, but the intravenous administration is more effective in limiting visceral pathology.

Finally, to assess the translational potential, the authors tested in utero ultrasound-guided delivery of AAV9-GFP vectors to two midgestation fetal macaques. Both macaques presented normally at birth and there was extensive GFP expression in all structures of the brain.

Waddington highlights two almost separate points in this article. "First, nGD may be curable by gene therapy." Waddington and colleagues are working with Apollo Therapeutics to develop and optimize a vector that can be taken forward to the clinic for postnatal gene therapy. "Second, fetal gene therapy is technically possible (when medically justifiable) and maybe even an improvement on postnatal gene therapy," he says. Fetal gene therapy, however, might be a lot harder to move forward as clinical trials in pregnant women present many challenges.

\section{Teresa Villanueva}

ORIGINAL ARTICLE Massaro, G. et al. Fetal gene therapy for neurodegenerative disease of infants. Nat. Med. https://doi.org/10.1038/ s41591-018-0106-7 (2018) 